\title{
DELAYED TRAUMATIC TETRAPLEGIA
}

\author{
P. J. SCott, ExeTER, England
}

In some patients with traumatic tetraplegia no radiological evidence of fracture or dislocation of the cervical spine is found. If myelography or necropsy fails to reveal protrusion of an intervertebral disc the mechanism of the cord injury may remain a mystery.

At one time it was believed that hyperflexion caused dislocation of the posterior intervertebral joints, with contusion of the cord by the neural arch at the moment of injury, and that spontaneous reduction accounted for the subsequent normal radiographic appearances. This theory was criticised by Cramer and McGowan (1944), and Barnes (1948) showed that a posterior dislocation in flexion that was capable of spontaneous reduction did not produce sufficient narrowing of the spinal canal to impinge on the cord. Furthermore, Roaf (1960) was unable to produce a flexion dislocation of the posterior joints without also causing a crush fracture of a vertebral body.

In a few reported cases necropsy has revealed rupture of the anterior longitudinal ligament with a tear through an intervertebral disc. Barnes(1948) attributed this injury to a hyperextension dislocation, and suggested that the neural arch had contused the cord. Taylor and Blackwood (1948) reported a similar case and offered the same explanation. But Roaf (1960) was unable to rupture the anterior longitudinal ligament by hyperextension without also fracturing the neural arch. A tear through the anterior longitudinal ligament and disc, without fracture, could be produced only by rotational or shearing forces, just as dislocation without fracture could only be produced by rotational force. This suggests that a transient displacement, with a tear of the anterior longitudinal ligament and disc, could occur only with a rotational or shearing force, and that it is by scissoring that a direct contusion of the cord may be produced.

There remain those cases in which no fracture, no dislocation and no tear of the anterior longitudinal ligament or disc have been found. Crooks and Birkett (1944) and Barnes (1948) drew attention to the frequency of this type of traumatic tetraplegia in older patients with arthritis of the cervical spine. Barnes tentatively suggested that cord contusion might result from simple hyperextension of a spinal cord already narrowed by osteophytes and by the compensatory cervical lordosis. Taylor (1951) reported a patient in whom the segmental scar in the cord was more extensive in the dorsal part, and suggested that the ligamentum flavum, buckled inwards by hyperextension, had contused the cord. Roaf (1960) found that, if the disc turgor was impaired, rotational subluxation of the cervical spine could occur, without rupture of the anterior longitudinal ligament, and put this forward to explain the mechanism.

These theories are based on the assumption that the spinal cord was damaged at the moment of injury. This assumption is in turn based on the usual finding of the greatest loss of cord function at the time the patient is first examined, although this is seldom immediately after injury. However, in some patients with traumatic tetraplegia who also have ankylosing spondylitis the loss of cord function has increased after the initial examination (Barnes 1962); and in the patient reported here the tetraplegia was not only progressive but also delayed in onset.

\section{CASE REPORT}

A fifty-four-year-old man, who had received radiotherapy for ankylosing spondylitis at the age of forty-one, sustained a single blow from a fist on the side of the jaw, and fell backwards to the floor, striking the top of his head on a low footrail. 
He was assisted to his feet, walked three hundred yards to a bus stop, and subsequently walked a quarter of a mile from another bus stop, arriving home an hour and a half after the blow had been struck. On reaching home he complained of pain down both his arms and

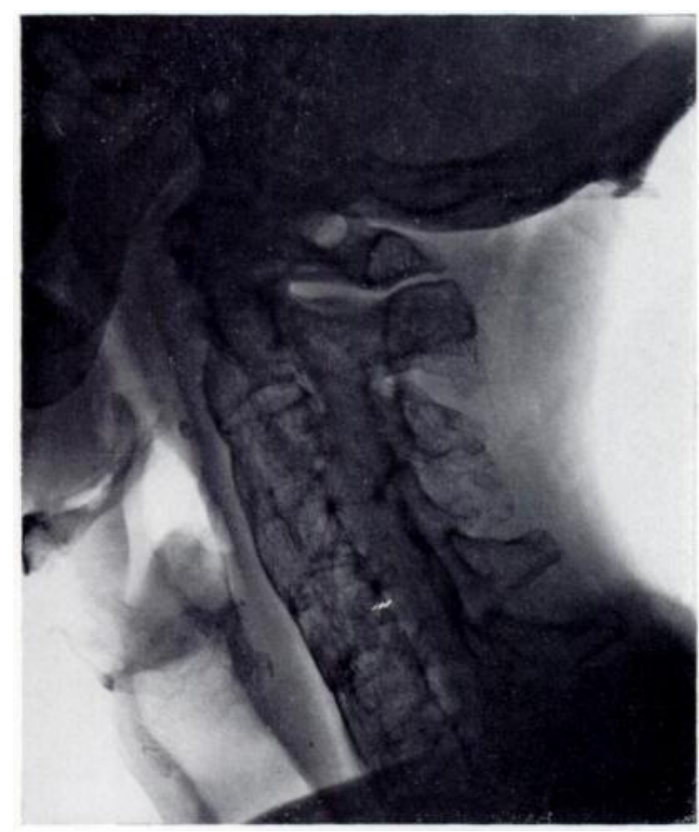

FIG. 1

A lateral view of the cervical spine after the injury. went to bed. He was first examined two hours after the injury and was then unable to move his legs, or to feel pain in them.

Twenty-four hours later there was complete paralysis up to, and including, the fingers, with loss of pain sensation from the level of the seventh and eighth cervical segment. Touch and position sense were retained, but over the next few days the sensory loss became complete. No subsequent recovery occurred.

There was a bruise on the side of the chin and an abrasion on the vertex. The spine was straight, with no movement below the axis. No radiological evidence of spinal fracture or dislocation was found, but there were advanced changes of ankylosing spondylitis (Fig. 1). The cerebrospinal fluid was clear, and manometry was normal with a free rise and fall: myelography showed no obstruction to the passage of iodised oil.

The patient died a year after injury. Necropsy revealed no injury of the ankylosed vertebral column and no irregularity of the spinal canal. At the level of the fifth cervical vertebra there was softening and microscopic disorganisation of the spinal cord, with degeneration of the ascending tracts above and the descending tracts below. At the same level there was an area of old blood-staining on the outer surface of the dura mater.

\section{CONCLUSI $2: \cdots$}

The delayed onset of tetraplegia in this patient cannot be explained on the basis of contusion of the cord at the moment of injury; nor does the initial sparing of touch and proprioceptive sensation agree with the theory of cord contusion by the neural arch or ligamentum flavum in hyperextension; nor does it seem likely that any transient displacement can have occurred in a rigid, undamaged, spine.

The progress was consistent with increasing compression of the cord, but normal spinal manometry and myelography, and absence of necropsy evidence of cord compression, are against this.

The possibility of haemorrhage into the cord is largely discounted by the delayed onset of paralysis, the absence of blood in the cerebrospinal fluid, and the absence of evidence of intrathecal haemorrhage at necropsy.

It is suggested that the condition can only be satisfactorily explained by progressive ischaemia and infarction of a segment of the cord because of arterial injury. The finding of old blood-staining on the outer surface of the dura at the necropsy is evidence of extradural vascular injury.

Whether or not the presence of ankylosing spondylitis, or merely of atherosclerosis, renders the arterial supply of the spinal cord vulnerable to contusion and thrombosis is a matter of speculation, but spinal cord infarction could explain the reported disparity between 
the loss of spinal cord function and the severity of vertebral column injury in older patients with cervical spines already the seat of disease.

\section{SUMMARY}

1. The theories that have been advanced to explain the occurrence of traumatic tetraplegia in patients without evidence of vertebral column injury are reviewed.

2. Traumatic tetraplegia of delayed onset is described in a middle-aged man with ankylosing spondylitis. There was no injury of the vertebral column.

3. The reasons are given for suggesting that the tetraplegia was caused by injury to the arterial supply of the cord.

I wish to thank Mr Norman Capener for permission to report this case.

\section{REFERENCES}

Barnes, R. (1948): Paraplegia in Cervical Spine Injuries. Journal of Bone and Joint Surgery, 30-B, 234.

BARnes, R. (1962): Personal communication.

Cramer, F., and McGowan, F. J. (1944): The Role of the Nucleus Pulposus in the Pathogenesis of So Called "Recoil" Injuries of the Spinal Cord. Surgery, Gynecology and Obstetrics, 79, 516.

Crooks, F., and BirketT, A. N. (1944): Fractures and Dislocations of the Cervical Spine. British Journal of Surgery, 31, 252.

Roaf, R. (1960): A Study of the Mechanics of Spinal Injuries. Journal of Bone and Joint Surgery, 42-B, 810.

TAYLOR, A. R. (1951): The Mechanism of Injury to the Spinal Cord in the Neck Without Damage to the Vertebral Column. Journal of Bone and Joint Surgery, 33-B, 543.

TAYLOR, A. R., and BlaCKwOOD, W. (1948): Paraplegia in Hyperextension Cervical Injuries with Normal Radiographic Appearances. Journal of Bone and Joint Surgery, 30-B, 245. 